\title{
ETUDE DES POSSIBILITÉS DE TRANSFERT AUTOMNAL EN MER DE JUVENILES DE TRUITE FARIO (SALMO TRUTTA)
}

\author{
Edwige QUILLET*, Francine KRIEG** A. HAPPE*, B. CHEVASSUS** \\ * I.F.R.E.M.E.R. Centre de BREST, B.P. 337, 29273 - BREST Cedex, France \\ ** I.N.R.A., Laboratoire de Génétique des Poissons, 78350 - JOUY-en-JOSAS, France
}

Reçu le 28 Février 1986

Accepté le 24 Septembre 1986

Received 28 February, 1986

Accepted 24 September, 1986

\section{RÉSUMÉ}

Deux modes de transfert automnal en mer (direct ou progressif) ont été testés chez la truite commune (Salmo trutta) sur des poissons âgés de 10 mois et deux catégories de poids différentes. ( $:$ Pm $=40 \mathrm{~g}, \mathrm{~S}: \mathrm{Pm}=50 \mathrm{~g}$ ).

Au cours des trois premières semaines qui suivent le transfert, une mortalité relativement élevée (22\%) est observée sur le lot "I" transféré directement, les trois autres lots (I et S transférés progressivement, $\mathrm{S}$ transféré directement) présentant une bonne survie (97\%). Cette mortalité affecte essentiellement les plus petits individus (inférieurs à 40 grammes). Au cours de la période d'observation ultérieure ( 5 mois), les survies sont élevées et comparables dans les quatre. lots. A l'issue de cette période, les poids moyens des lots ne diffèrent pas selon le mode de transfert et sont en outre supérieurs à ceux des témoins de même taille initiale, conservés en eau douce. II apparaît donc possible d'effectuer un transfert automnal direct en mer pour des animaux supérieurs à 40 grammes sans effets néfastes sur leur survie et leur croissance ultérieure.

\section{STUDY OF POSSIBILITIES OF AUTUMNAL SEAWATER TRANSFER OF BROWN TROUT FINGERLINGS (SALMO TRUTTA)}

\section{SUMMARY}

Two ways of adaptation (direct or progressive) to $35 \%$ salinity seawater were investigated on brown trout ten months old for two different classes of mean weight $(\mathrm{I}: \mathrm{Wm}=40 \mathrm{~g} ; \mathrm{S}: \mathrm{Wm}=50 \mathrm{~g})$.

During the first three weeks after transfer into seawater, significant mortality (22\%) was observed in "I" group transferred directly in seawater while good survival was exhibited by the three other groups (97\%) (I and S with progressive transfer,S directly transferred). During the following period (5 months), survivals were good and similar in the four groups. At the end of this period, no difference in mean weight was detected between the two ways of transfer into seawater and mean weight was higher than for the control group in freshwater. So direct transfer, whithout negative effects on survival and growth, appears possible for individuals bigger than $40 \mathrm{~g}$.

\section{INTRODUCTION}

Actuellement, en France, l'aquaculture en mer des salmonidés est príncipalement basée sur l'élevage en cycle court (Novembre-Juin) de la truite arc-en-ciel, ce cycle étant essentiellement imposé par la faible survie estivale de cette espèce sur le littoral français. Afin de développer des cycles de production plus longs ou réalisés à d'autres périodes, l'élevage d'autres espèces de salmononidés a été envisagé. Parmi elles, la truite commune (Salmo trutta) montre une bonne survie estivale (supérieure à $80 \%$, QUILLET et al. (1985), et les performances de croissance observées permettent d'envisager la production d'animaux de l'ordre du kilo à l'issue du premier été en mer, c'est-à-dire à l'âge de 22 mois. De ce fait, l'élevage de la truite fario pourrait constituer une production cornplémentaire de celle de la truite arc-en-ciel concentrée sur le printemps. Jusqu'à présent, les transferts en mer de cette espèce ont été réalisés avec succès au printemps sur des animaux $1^{+}$d'un poids $^{\prime}$ supérieur à $60 \mathrm{~g}$ (BCEUF et HARACHE, 1982 ; 1984 ; QUILLET et al., 1985). Cependant, afin de limiter la durée d'élevage en eau douce (cette espèce y étant plus difficile à élever que l'arc-en-ciel et présentant un taux de croissance des juvéniles plus faible), nous avons testé la possibilité de réaliser un transfert automnal d'animaux âgés de moins d'un an. Dans cette optique, nous avons tenté :

- d'une part, de préciser les tailles limites de transfert en constituant des groupes de poids moyens différents,

- d'autre part, d'évaluer les conséquences à court et moyen terme des modalités de transfert en mer en comparant un transfert direct à $35 \%$ de salinité à un transfert progressif (augmentation graduelle de la salinité). 


\section{MATERIEL ET METHODES}

\section{1) Matériel animal}

Les animaux, élevés dans la pisciculture de l'INRA à Gournay-sur-Aronde (Oise), représentent un mélange de cinq souches issues de piscicultures différentes. Deux groupes, triés sur le poids, sont constitués à l'âge de dix mois : un lot de tête (notéS) de poids moyen $30 \mathrm{~g}$ et un groupe plus petit (notél) de poids moyen $20 \mathrm{~g}$. Ces animaux sont ensuite transportés au Centre de l'IFREMER à Brest et placés dans des bacs d'élevage de $2 \mathrm{~m}^{3} \mathrm{a}$ une température moyenne de $10^{\circ} \mathrm{C}$. Après une période d'adaptation de 40 jours, les lots ont atteint les poids moyens de $39,6 \mathrm{~g}$ (I) et $48,6 \mathrm{~g}$ (S). Chacun de ces lots est alors divisé en trois groupes destinés au transfert direct (D), au transfert prugressif (P) et à la constitution d'un petit lot témoin conservé en eau douce. Les effectifs des divers groupes ainsi constitués sont donnés dans le tableau 1.

\section{2) Protocole de transfert}

Le protocole des transferts directs et progressifs est détaillé dans le tableau 1.

Les lots SP et IP sont transférés progressivement (les modalités d'élévation de la salinité sont précisées dans le tableau 2).

Après seize jours à une salinité de $35 \%$, les animaux sont transportés à la SEMII (Station Expérimentale Marine INRA-IFREMER basée à Camaret) et, après marquage par ablation de nageoires (adipeuse et/ou pelvienne), sont regroupés dans une cage de $60 \mathrm{~m}^{3}$. Les lots témoins conservés en eau douce sont éliminés, la comparaison des performances en eau douce et en eau de mer faisant l'objet d'une expérimentation à plus grande échelle non présentée ici.

\begin{tabular}{|c|c|c|c|}
\hline & TRANSFERT PROGRESSIF & TRANSFERT DIRECT & EAU DOUCE \\
\hline LOT & $\begin{array}{cc}S & I \\
146 & 159\end{array}$ & $\begin{array}{cc}S & I \\
152 & 138\end{array}$ & $\begin{array}{rr}S & 1 \\
34 & 36\end{array}$ \\
\hline Le $23 / 11$ & $\begin{array}{l}\text { Début du transfert } \\
\text { progressif } \\
\text { Etude des paramètres } \\
\text { sanguins (E.P.S.) }\end{array}$ & & E.P.S. \\
\hline Le $23 / 12$ & $\begin{array}{l}48 \text { h à } 31 \% \text { de salinité } \\
\text { (E.P.S) } \\
\text { Passage à } 35 \%\end{array}$ & $\begin{array}{c}\text { Transfert direct à } \\
35 \% \%\end{array}$ & E.P.S. \\
\hline Le $05 / 12$ & $\begin{array}{c}48 \text { h à } 35 \% \text { de salinité } \\
\text { (E.P.S.) }\end{array}$ & $\begin{array}{c}48 \text { h à } 35 \% \text { de sal inité } \\
\text { (E.P.S.) }\end{array}$ & E.P.S. \\
\hline Le $18 / 12$ & $\begin{array}{r}16 \text { jours à } 35 \% \\
\text { (E.P.S.) } \\
\text { Transport à La sta } \\
\text { de Camare }\end{array}$ & $\begin{array}{l}\text { de salinité } \\
\text { tion expérimentale } \\
t \text { s/Mer }\end{array}$ & $\begin{array}{l}\text {.E.P.S. } \\
\text { Fin de L'experience }\end{array}$ \\
\hline
\end{tabular}

Tableau 1 : Protocole experimental.

Table 1 : Experimental protocol. 


\begin{tabular}{|c|c|c|c|c|c|c|c|}
\hline DATE & $22 / 11$ & $23 / 11$ & $26 / 11$ & $27 / 11$ & $29 / 11$ & $01 / 12$ & $03 / 192$ \\
\hline SALINITE & E.D. & $15 \%$ & $20 \%$ & $25 \%$ & $28 \%$ & $31 \%$ & $35 \%$ \\
\hline
\end{tabular}

Tableau 2 : Evolution de la salinité pendant le transfert progressif.

Table 2 : Evolution of salinity during progressive transfer.

\section{3) Paramètres étudiés}

\section{a) La survie}

Elle est étudiée sur quatre périodes :

- pendant le transfert progressif,

- au cours des 16 jours qui suivent le transfert à $35 \%$. Pendant cette période, les poissons morts sont mesurés et comparés à la moyenne du lot dont ils sont issus.

- dans la période qui suit le transport à la SEMII,

- au cours des cinq mois d'élevage en cage (janvier à mai).

\section{b) Les paramètres sanguins}

Plusieurs auteurs ont montré sur différentes espèces de salmonidés (la truite arc-en-ciel, la truite commune, l'omble de fontaine, le saumon coho) qu'il existe une bonne relation entre les performances du poisson à long terme et les modifications ioniques du plasma dans les semaines qui suivent le transfert en mer (LAHLOU et al., 1975 ; BCEUF et al., 1982 ; BCEUF et HARACHE, 19821984 ; JOHNSTON et CHEVERIE, 1985). On peut donc considérer l'évolution des paramètres sanguins comme un outil efficace de prédiction des performances à long terme.

Des prélèvements de sang (aorte postérieure) sont donc effectués sur douze animaux (deux réplicats de six individus) aux salinités suivantes : $0 \%$, 48 heures après $31 \%$, et 16 jours après $35 \%$ (tous les lots). Des prélèvements sont effectués sur les lots témoins restés en eau douce au début du transfert progressif, à la date du transfert direct et 16 jours après ce dernier. Les échantillons de sang sont centrifugés ; le plasma recueilli est stocké au congélateur jusqu'à l'analyse. Les mesures de pression osmotique du plasma sont faites sur microosmomètre Roebling et le dosage des ions $\mathrm{Cl}^{-}$sur chloridomètre Radiometer selon les techniques décrites dans BFUF et al., (1978a, b). Les résultats sont exprimés par la moyenne arithmétique des deux réplicats.

\section{c) La croissance}

Elle est mesurée en eau douce avant le transfert, puis mensuellement en mer.

\section{RÉSULTATS}

\section{1) Survie}

- Montée en salinité (transfert progressif).

Aucune mortalité n'est observée pendant la montée en salinité. Les résultats ultérieurs sont résumés dans la figure 1 .

- 16 jours après le stade $35 \%$ de salinité (transfert direct ou progressif).

Alors que les trois lots IP, SP, SD présentent tous une survie très élevée au cours de cette période $(97 \%)$, les mortalités du lot ID s'élèvent à $22 \%$. En outre, dans ce dernier groupe, la moitié de la mortalité se produit dans les $48 \mathrm{~h}$ qui suivent le transfert en mer et affecte essentiellement les poissons les plus petits (les individus de moins de $150 \mathrm{~mm}$ représentent environ $90 \%$ des mortalités, fig. 2). Il faut noter également que dans les trois autres groupes (IP, SP, SD) la mortalité bien que très faible concerne les mêmes individus.

- Dans le mois suivant le transport à Camaret.

Tous les groupes présentent une légère mortalité $(7 \%)$, cette dernière étant équivalente dans les divers lots, quel que soit le mode de transfert pratiqué.

- A long terme.

On ne note aucune différence significative entre les lots. Tous présentent une excellente survie (95 à $100 \%$ ). 


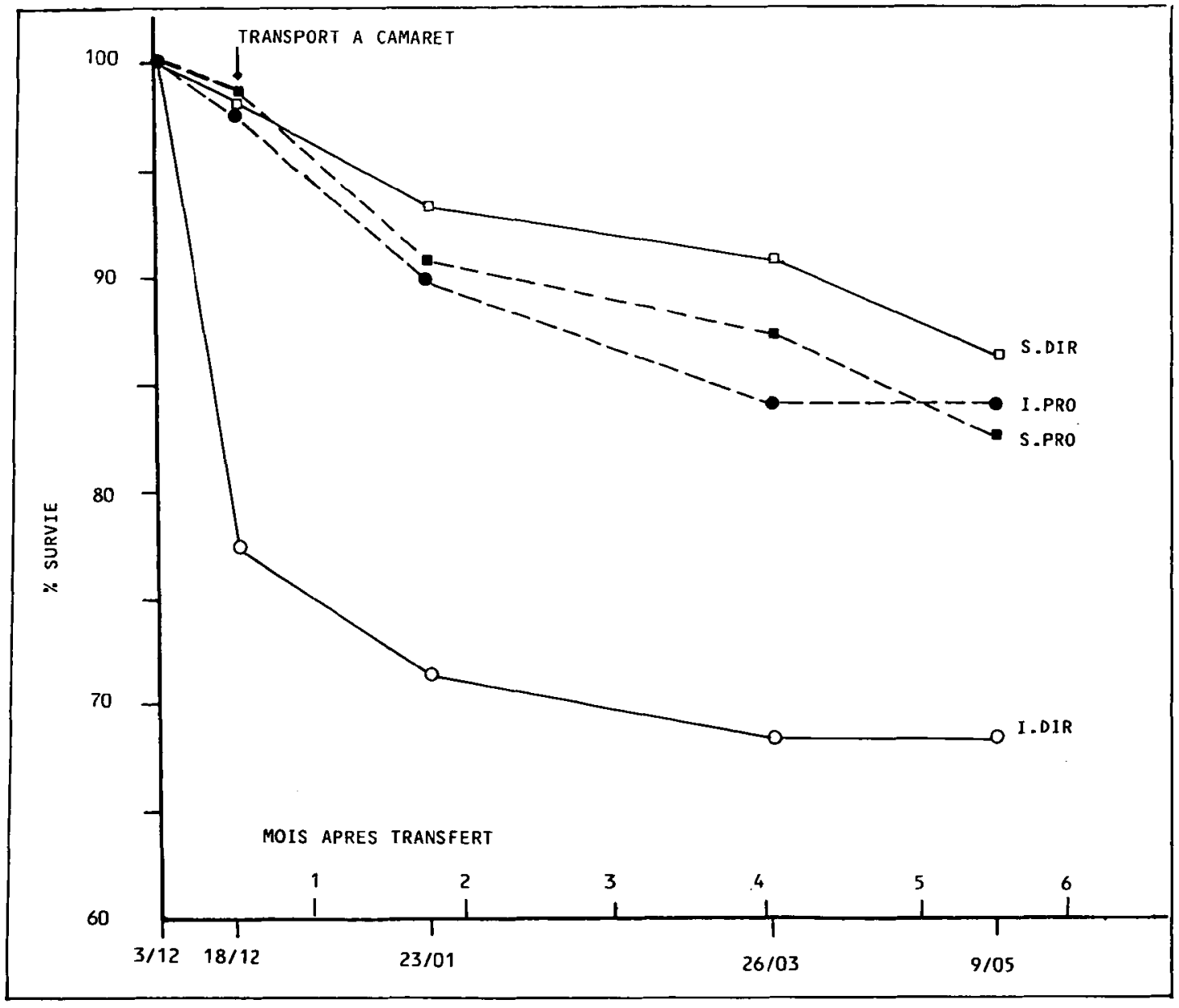

Figure 1 : Survie des quatre groupes après transfert en mer.

Figure 1 : Survival of the four groups following seawater transfer.

\section{2) Analyse du plasma sanguin}

Les résultats sont regroupés dans le tablẹau 3 et la figure 3. Les valeurs de pression osmotique et de concentration d'ions $\mathrm{Cl}^{-}$restant constantes sur toute la période d'étude, les résultats des trois prélèvements effectués sur les animaux conservés en eau douce ont été regroupés.

Dans le cas du transfert progressif (lots SP et IP), on observe à $31 \%$ de salinité une légère augmentation de la pression osmotique (SP : $+9.9 \%, \mathrm{IP}:+11,4 \%$ ) et de la concentration en ions $\mathrm{Cl}^{-}$ (SP : $+17,1 \%$, IP : + 14,2\%) par rapport au témoin eau douce, valeurs qui continuent à augmenter lorsque la salinité atteint $35 \%$ (SP : $+14,4 \%$, IP : + 12,6\% (pression osmotique), SP : $20,9 \%$, IP : $+15 \%$ (chlorémiel). Après seize jours eñ mer, les valeurs de pression osmotique et la chlorémie se stabilisent à environ $+10 \%$ du niveau observé en eau douce.

Les lots transférés directement en mer (SD, ID) montrent initialement une augmentation beaucoup plus forte de la pression osmotique (SD : + 35\%, ID : + 37\%) et de la concentration en ions $\mathrm{Cl}^{-}$(SD : + $40 \%$, ID : + $47 \%$ ). Les valeurs moyennes après 16 jours à $35 \%$ de salinité sont cependant à peine supérieures à celles observées en eau douce et de même ordre que celles observées pour les lots transférés progressivement. Sur la base de ces résultats il semble d'autre part que le niveau de déséquilibre osmotique ne soit pas fonction de la classe de taille étudiée, les valeurs de pression osmotique et de concentration en ion $\mathrm{Cl}^{-}$n'étant pas significativement différentes dans les lots I et $\mathbf{S}$.

L'analyse des corrélations entre le poids et les valeurs de pression osmotique et de chlorémie mesurées par individu confirme également l'absence d'une telle relation. 

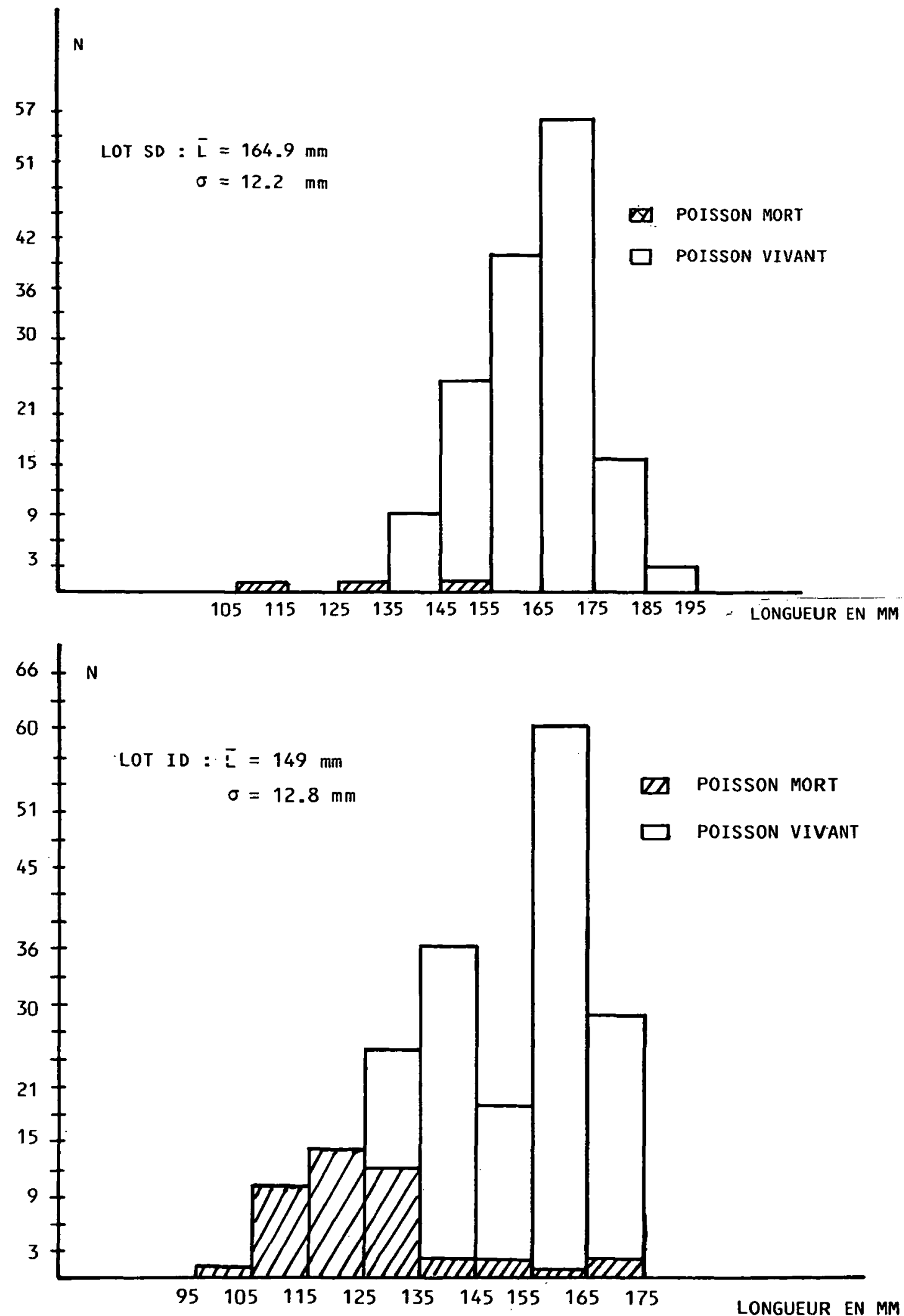

Figure 2 : Répartition des morts en fonction de la taille dans les groupes ID et SD durant les seize jours après le transfert en mer.

Figure 2 : Length distribution of deadfish in ID and SD groups during the 16 days following seawater transfer. 


\begin{tabular}{|c|c|c|c|c|c|c|c|c|c|c|}
\hline & Eau douce & $\begin{array}{c}48 \mathrm{~h} \\
\text { Transfert } \\
\mathrm{s}\end{array}$ & $\begin{array}{c}31 \% \\
\text { progressif } \\
I\end{array}$ & $\begin{array}{c}\text { Transfer } \\
\mathrm{s}\end{array}$ & $\begin{array}{c}48 \text { h à } 35 \\
\text { t progress if } \\
\text { I }\end{array}$ & $\begin{array}{l}\text { Transfert direct } \\
\mathrm{s}\end{array}$ & $\begin{array}{c}\text { Transfer } \\
s\end{array}$ & $\begin{array}{c}16 \mathrm{j} \text { à } 35 \\
\text { progressif } \\
\mathrm{I}\end{array}$ & $\begin{array}{l}\% \\
\begin{array}{c}\text { Iransfert } \\
5\end{array}\end{array}$ & direct \\
\hline POIDS & $\begin{array}{r}45,9 \\
\pm \quad 3,4\end{array}$ & $\begin{array}{r}52,34 \\
\pm \quad 4,6\end{array}$ & $\begin{array}{l}45,58 \\
\pm 9,3\end{array}$ & $\begin{array}{r}49 \\
\pm \quad 6,5\end{array}$ & $\begin{array}{r}46,8 \\
\pm \quad 10,4\end{array}$ & $\begin{array}{r}48,6 \quad 35,4 \\
\pm 7,8 \pm 7,7\end{array}$ & $\begin{array}{r}57,2 \\
\pm \quad 6,7\end{array}$ & $\begin{array}{r}48,2 \\
\pm \quad 7,0\end{array}$ & $\begin{array}{r}52,8 \\
\pm \quad 8,5\end{array}$ & $\begin{array}{r}47,6 \\
+\quad 3,9\end{array}$ \\
\hline $\begin{array}{l}\text { PRESSION } \\
\text { OSMOT IQUE } \\
\text { P.O. }\end{array}$ & $\begin{array}{l}288,4 \\
\pm 3,9\end{array}$ & $\begin{array}{c}321 \\
\pm 11,1\end{array}$ & $\begin{array}{l}316,5 \\
\pm 6,7\end{array}$ & $\begin{array}{r}330 \\
\pm 10,1\end{array}$ & $\begin{array}{r}324,7 \\
\pm 10,5\end{array}$ & $\begin{array}{cc}388 & 394,8 \\
\pm 19,2 & \pm 25\end{array}$ & $\begin{array}{l}322 \\
\pm 7,9\end{array}$ & $\begin{array}{r}323,6 \\
\pm 20,1\end{array}$ & $\begin{array}{l}317,7 \\
\pm 9,55\end{array}$ & $\begin{array}{r}310,2 \\
\pm 16,6\end{array}$ \\
\hline $\begin{array}{l}\text { CONCENTRATION } \\
\text { EN IONS } \\
\text { CL.- }^{-}\end{array}$ & $\begin{array}{l}122,6 \\
\pm 2,39\end{array}$ & $\begin{array}{l}143,6 \\
\pm 6,6\end{array}$ & $\begin{array}{l}140,1 \\
\pm 4,4\end{array}$ & $\begin{array}{r}148,3 \\
\pm 5,4\end{array}$ & $\begin{array}{r}140,9 \\
\pm 8,3\end{array}$ & $\begin{array}{r}169,8 \quad 180,4 \\
\pm 9,1 \pm 10,1\end{array}$ & $\begin{array}{l}134,1 \\
\pm 4,9\end{array}$ & $\begin{array}{l}137,6 \\
\pm 4,9\end{array}$ & $\begin{array}{l}139,4 \\
\pm 7\end{array}$ & $\begin{array}{l}137,5 \\
\pm 8,1\end{array}$ \\
\hline $\begin{array}{l}\text { CORRELAT ION } \\
\text { POIDS/P.O. }\end{array}$ & 0,32 & \multicolumn{2}{|c|}{0,09} & \multicolumn{2}{|c|}{$-0,34$} & $-0,3$ & \multicolumn{2}{|c|}{$-0,19$} & \multicolumn{2}{|c|}{$-0,02$} \\
\hline $\begin{array}{l}\text { CORRELATION } \\
\text { POIDS/ } / \mathrm{CL}^{-}\end{array}$ & 0,25 & \multicolumn{2}{|l|}{$-0,1$} & \multicolumn{2}{|c|}{$-0,35$} & $-0,41$ & \multicolumn{2}{|c|}{$-0,09$} & \multicolumn{2}{|c|}{$-0,23$} \\
\hline
\end{tabular}

Moyenne \pm écart type de la moyenne.

Tableau 3 : Mesure de la P.O. et de la concentration en ions $\mathrm{Cl}^{-}$en fonction de la salinité. Corrélation poids/P.O. et poids/CI-.

Table 3 : Measure of plasma osmotic pressure and ionic concentration $\mathrm{Cl}^{-}$with increasing salinity. Correlation between weight and $\mathrm{P} . \mathrm{O}$. weight and $\mathrm{Cl}^{-}$.

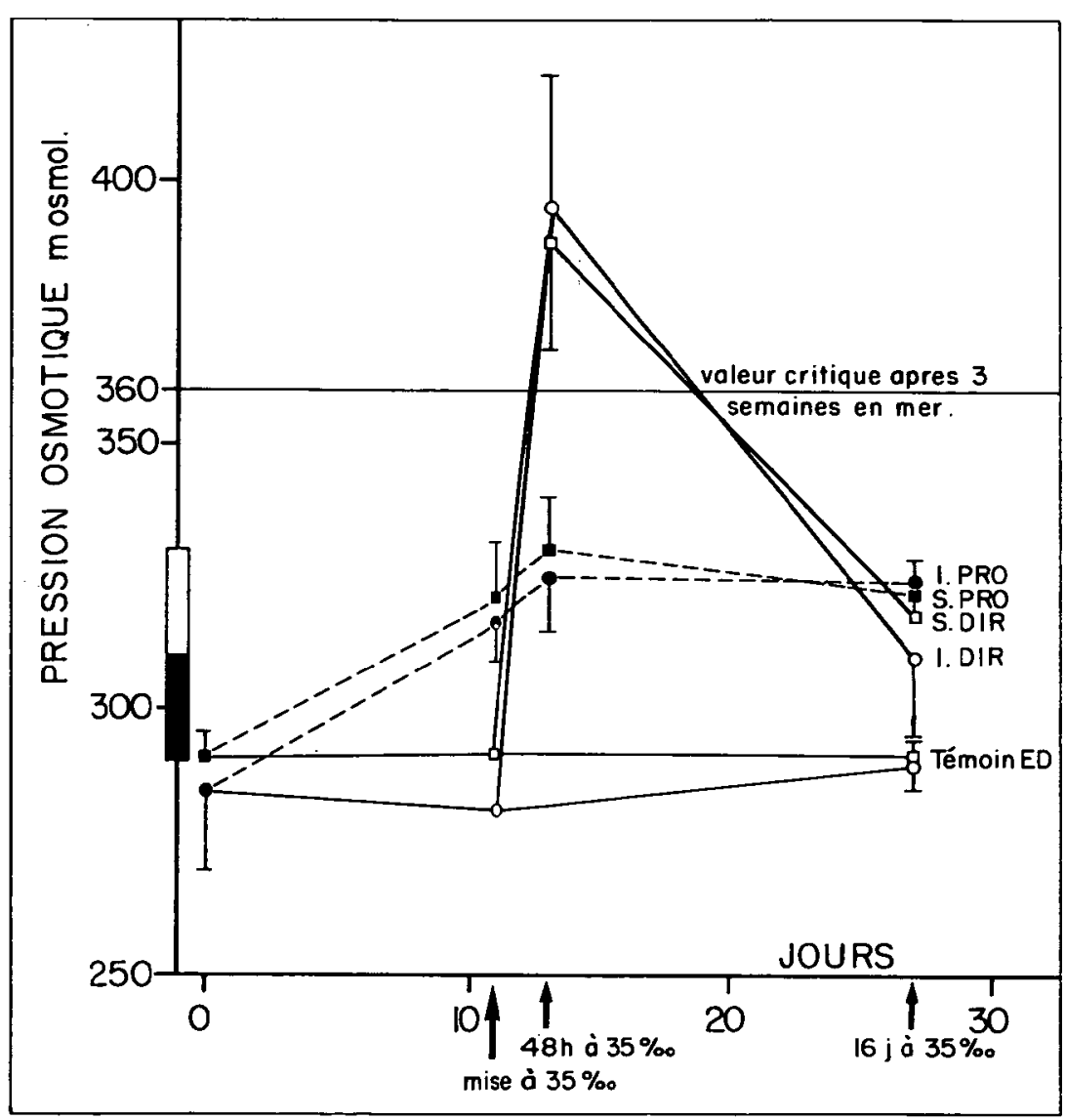

Domaine de variation

en eau de mer

en eau douce

Figure 3 : Evolution de la pression osmotique après transfert en mer.

Figure 3 : Evolution of the plasma osmotic pressure following seawater transfer. 


\section{3) Croissance}

Les croissances observées dans les divers lots sont schématisées dans la figure 4. Cette courbe présente également à titre indicatif la croissance observée sur un témoin conservé dans une pisciculture en eau douce (Station INRA de Gournay-sur-Aronde).

Tous les groupes accusent un ralentissement marqué de la croissance dans la période qui suit le transfert en mer, celui-ci étant cependant particulièremént net pour les lots ayant subi un transfert direct. Ainsi, les taux de croissance journaliers représentent respectivement $45 \%$ (SD), $30 \%$ (ID), $75 \%$ (SP) et $46 \%$ (IP) des taux de croissance observées en eau douce dans la période précédant le transfert. II convient cependant de noter que ce ralentissement est le résultat d'un effet conjugué du transfert en mer en lui-même et d'autres facteurs (transport dans les cages, adaptation à un nouveau type d'élevage, passage d'un bassin à une cage en filet) également susceptibles d'y contribuer.

Au cours du deuxième mois en mer, on constate une reprise de la croissance. L'évolution relative des divers groupes conduit au terme de 5 mois d'élevage à une disparition des écarts de poids consécutifs au mode de transfert. En outre, à cette date le poids des animaux élevés en mer apparaît supérieur à celui des lots conservés en eau douce à la pisciculture de Gournay-sur-Aronde $(+15 \%$ dans le cas des lots $\mathrm{S},+20 \%$ pour les lots I).

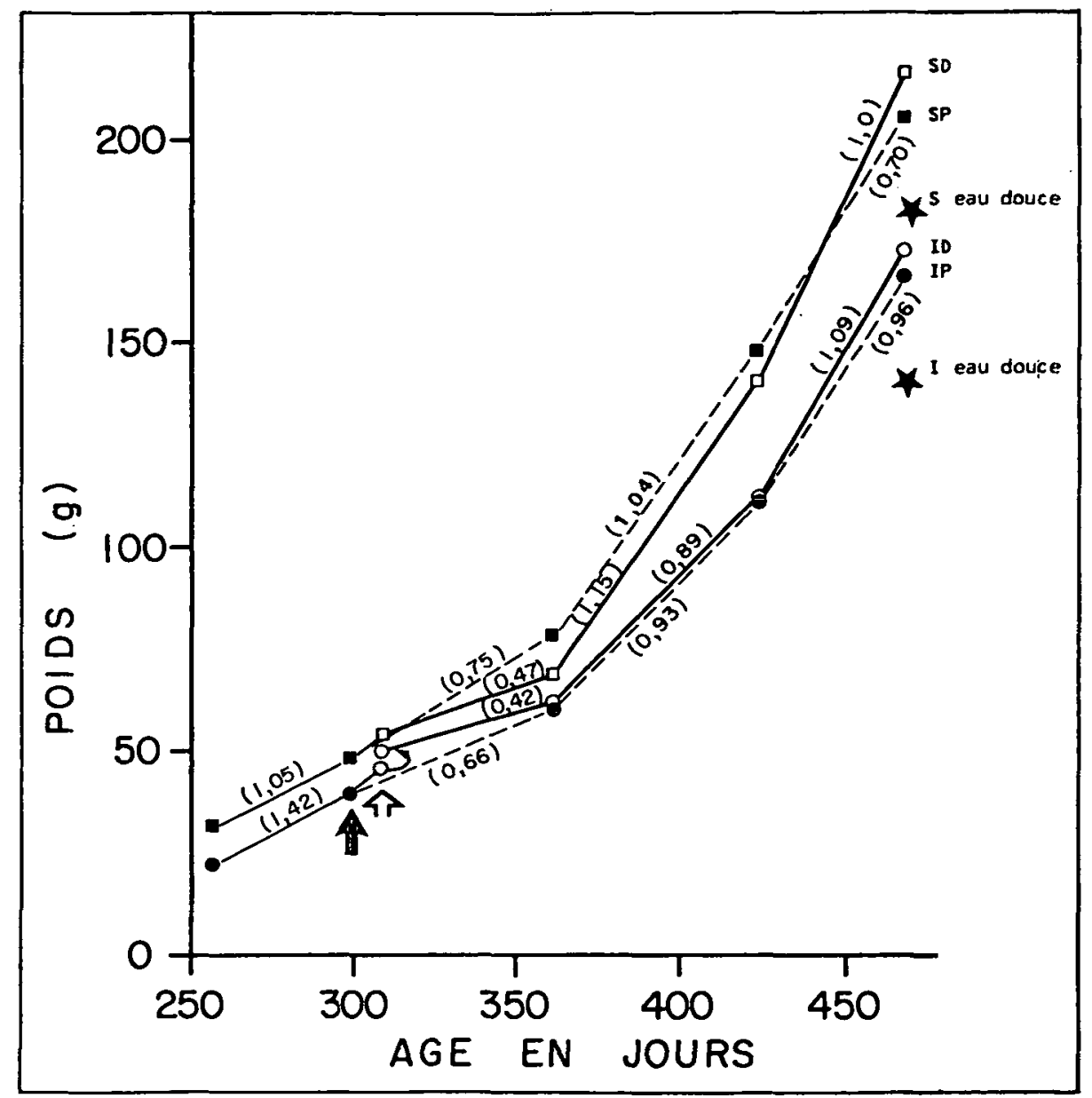

: Correction liée à la mortalité sélective au transfert.

: Début transfert progressif

: Fin transfert progressif et transfert direct

$(1,05)$ Taux de croissance. spécifique (en $z$ par jour)

$$
\begin{aligned}
& S: P_{m}=50 \mathrm{~g} \text { à la mise en mer } \\
& I: P_{m}=40 \mathrm{~g} \text { à la mise en mer }
\end{aligned}
$$

Figure 4 : Croissance des différents groupes.

Figure 4 : Growth of the different groups. 


\section{DISCUSSION ET CONCLUSIONS}

\section{- Transfert progressif}

L'analyse des caractéristiques du plasma sanguin révèle dans ce cas que les poissons s'adaptent rapidement à leur milieu et restent pratiquement toujours dans une gamme "normale", pour l'eau de mer, de variation du niveau de pression osmotique (290-330 mosm) et de la concentration des ions chlorure. Ces résultats sont en accord avec la survie excellente observée quel que soit le poids moyen du lot considéré (les poissons les plus petits faisant environ 20-25 g), et avec le ralentissement de croissance relativement modéré constaté dans la période qui suit le transfert en mer.

\section{- Transfert direct}

On observe une nette différence de comportement entre les deux groupes testés. Alors que la mortalité est très faible dans le lot $S(\mathrm{Pm}$ initial $50 \mathrm{~g})$, une proportion non négligeable des poissons meurt dans le groupe I ( $\mathrm{Pm}$ initial $40 \mathrm{~g})$, cette mortalité affectant essentiellement les individus les plus petits (de poids inférieur à 30-35 grammes).

Ces résultats ne sont que partiellement en accord avec les données issues des analyses plasmatiques. En effet, sur les espèces ne smoltifiant pas, l'importance du déséquilibre de la balance hydrominérale d'un individu semble bien corrélée avec son poids (PARRY, 1960). Or, en valeur moyenne nous n'observons pas de différence de niveau de pression osmotique et de concentration d'ions $\mathrm{Cl}^{-}$entre les groupes de poids moyens différents. De même, nous n'avons pas trouvé de corrélation entre le poids et la pression osmotique, ni entre le poids et la concentration en ions $\mathrm{Cl}^{-}$ Plusieurs hypothèses peuvent expliquer en partie ces résultats :

\section{- La mortalité sélective :}

Les poissons les plus petits et donc présentant vraisemblablement un déséquilibre plus important meurent assez tôt. En effet la moitié de la mortalité du lot I s'est produite dans les 48 premières heures après le transfert, c'est-à-dire avant le premier prélèvement sanguin, pouvant ainsi biaiser les résultats.

\section{- L'hétérogénéité des souches.}

BCEUF et HARÁCHE (1982) ont comparé des truites fario issues d'une souche migratrice et d'autres issues d'une souche sédentaire. Après transfert en mer, ils ont observé une netteaugmentation de la P.O. chez les individus du lot sédentaire, contrairement aux animaux issus de migrateurs, les deux groupes présentant par ailleurs une excellente survie. Tous les animaux utilisés pour notre expérience proviennent de souches de pisciculture, ces souches étant considérées comme génétiquement peu différenciées (KRIEG et GUYOMARD, 1985). Cependant, il est possible que certaines d'entre elles puissent présenter des aptitudes plus marquées à la migration et ne révéler que de faibles déséquilibres osmotiques même chez des animaux de petite taillè.

Le ralentissement très brutal de la croissance peut être relié aux forts déséquilibres osmotiques observés à court terme ( $48 \mathrm{~h}$ ) et aux difficultés transitoires éprouvées par les poissons pour s'adapter à leur nouveau milieu. Le transport à Camaret peu de temps après le transfert en mer a sans doute contribué également à freiner la reprise de croissance en provoquant éventuellement un nouveau déséquilibre osmotique transitoire.

De cette étude, il ressort que malgré un déséquilibre physiologique important les poissons rétablissent leur balance hydrominérale très rapidement ( 15 jours). II faut cependant remarquer que dans le groupe I transféré directement, le freinage de croissance a pu être sous-estimé du fait de la mortalité sélective (affectant les individus les plus petits). Cependant, les performances des lots ID et IP sont similaires lors de la période 1-5 mois de mer lorsque les mortalités sont faibles. Aucun effet négatif à long terme n'affecte donc les'survivants.

Un transfert direct de truite fario dès le premier automne apparaît donc possible pour des animaux de plus de $40 \mathrm{~g}(13 \mathrm{à} 14 \mathrm{~cm})$. Cependant, ce seuil a été établi dans des conditions expérimentales précises et a priori "optimales", (période de récupération entre transport et transfert, faibles écarts thermiques entre l'eau douce et l'eau de mer) et à une période définie de l'année (décembre). II conviendrait donc d'analyser l'influence éventuelle de ces facteurs, et notamment d'un transfert réalisé immédiatement après le transport. Une étude plus précise de la notion de "poids limite" doit également être menée pour affiner nos premières conclusions. 


\section{REMERCIEMENTS}

Nous tenons à remercier G. BURGER, J.P. HISEUX (INRA), Y. NORMANT (IFREMER) et l'équipe travaillant à la station expérimentale (SEMII, Camaret) commune à ces deux organismes pour leur participation à l'élevage des animaux et au recueil des données présentées dans cet article.

Ces recherches ont fait l'objet d'un contrat cadre entre I'INRA et l'IFREMER.

\section{BIBLIOGRAPHIE}

BEEUF G., 1979. Contribution à l'étude de la smoltification et de l'adaptation à l'eau de mer chez le saumon coho, Oncorhynchus kisutch Walbaum, au cours de son développement. Thèse doctorat de $3^{\mathrm{e}}$ cycle. Université de Bordeaux I : $144 \mathrm{p}$.

BEUF G., HARACHE Y., 1982. Criteria for adaptation of salmonids to high salinity seawater in France.

Aquaculture, (28), 163-176.

BCEUF G., HARACHE Y., 1984. Adaptation osmotique à l'eau de mer de différentes espèces /Salmo trutta, Salmo gairdneri. Salvelinus fontinalis) et hybride (Salmo Trutta ${ }^{\circ} \times$ Salvelinus fontinalis $\sigma^{7} /$ de salmonidés. Aquaculture, (40), 343-358.

BCEUF G., LASSERRE P., HARACHE Y., 1978a. Problèmes posés par le métabolisme hydrominéral en aquaculture des salmonidés. Ichthyophysiologica Acta, 2: 44-49.

BCEUF G., LASSERRE P., HARACHE Y., 1978b. Osmotic adaptation of Oncorhynchus kisutch Walbaum. II - Plasma osmotic and ionic variations, and gill $\mathrm{Na}^{+}-\mathrm{K}^{+}$- adenosine triphosphatase activity of yearling coho salmon, transferred to sea-water. Aquaculture, 15(1), 35-52.

BEUF G., LE ROUX A., HARACHE Y., 1982. Indicateurs biochimiques de la préparation à la migration chez les salmonidés: Applications aux transferts d'eau douce en eau de mer chez les animaux d'aquaculture. Actes Colloque CNEXO $n^{\circ} 14$ Actual. Biochim. Mar. $n^{\circ} 5: 327-334$.

JOHNSTON C.E., CHEVERIE J.C., 1985. Comparative analysis of ionoregulation in rainbow trout (Salmo gairdneri) of different sizes following rapid and low salinity adaptation. Can. J. Fish. Aquat.Sci, 42 (12), 1994-2003.

KRIEG F., GUYOMARD R., 1985. Population genetics of French brown trout (Salmo trutta L) : Large geographical differentiation of wild populations and high similarity of domesticated stocks. Gen. Sel. Evol., 17 (2), 225-242.

LAHLOU B., CRENESSE D., BENSAHLA-TALET A., PORTHE-NIBELLE J., 1975. Adaptation de la truite d'élevage à l'eau de mer. Effets sur les concentrations plasmatiques, les échanges branchiaux et le transport intestinal du sodium. J. Physiol., (Paris), 70 : 593-603.

PARRY G., 1960. Development of salinity tolerance in the salmon (Salmo salar L.) and some related species. J. Exp. Biol., 37 : 425-434.

QUILLET E., CHEVASSUS B., KRIEG F., 1985. Bilan des études génétiques 1983-1985. S.E.M.I.I., document technique $n^{\circ} 2$. 